\title{
TRACKING CONTROL OF NONSMOOTH COMPLEMENTARITY LAGRANGIAN SYSTEMS
}

\author{
Jean-Matthieu Bourgeot, Bernard Brogliato \\ INRIA Rhône-Alpes, 655 avenue de l'Europe - 38330 Montbonnot, France \\ jean-matthieu.bourgeot@inrialpes.fr, bernard.brogliato@inrialpes.fr
}

Keywords: Hybrid mechanical system, unilateral constraints, asymptotic stabilty.

\begin{abstract}
In this paper we study the tracking control of Lagrangian systems subject to frictionless unilateral constraints. The stability analysis incorporates the hybrid and nonsmooth dynamical feature of the overall system. The difference between tracking control for unconstrained systems and unilaterally constrained ones, is explained in terms of closedloop desired trajectories and control signals. This work provides details on the conditions of existence of a controller which guarantees asymptotic stability.
\end{abstract}

\section{Introduction}

The focus of this paper is the tracking control of a class of nonsmooth fully actuated Lagrangian systems subject to frictionless unilateral constraints on the position. Such systems may a priori evolve in three different phases of motion : i) A free motion phase, ii) A permanently constrainted phase, iii) A transition phase whose goal is to stabilize the system on some constraint surface. With respect to the results in [3] [4] we give accurate conditions under which various types of stability are assured, which were missing in these references. For instance the $n$-degree-of-freedom case with $n \geq 2$ is solved in [3] only if a certain matrix is a Jacobian, which is quite restrictive as simple examples show $[2, \S 8.6]$. In [4] the existence of a specific transition phase closed-loop trajectory is assumed, without proof. These two points are addressed in this paper.

\subsection{Dynamics}

Let $X \in \mathbb{R}^{n}$ denote the vector of generalized coordinates. The systems we study in this paper are complementarity Lagrangian systems, with Lagrangian function $\mathcal{L}=$ $\frac{1}{2} \dot{X}^{T} M(X) \dot{X}-U(X)$, where $T(X, \dot{X})=\frac{1}{2} \dot{X}^{T} M(X) \dot{X}$ is the kinetic energy, $U(X)$ is the differentiable potential energy. The dynamics may be written as:

$$
\left\{\begin{array}{l}
M(X) \ddot{X}+C(X, \dot{X}) \dot{X}+G(X)=u+\nabla F(X) \lambda_{X} \\
F(X) \geq 0, \quad F(X)^{T} \lambda_{X}=0, \quad \lambda_{X} \geq 0 \\
\text { Collision rule }
\end{array}\right.
$$

where $M(X)=M^{T}(X) \in \mathbb{R}^{n \times n}$ is the positive definite inertia matrix, $F(X) \in \mathbb{R}^{m}$ represents the distance to the constraints, $\lambda_{X} \in \mathbb{R}^{m}$ are the Lagrangian multipliers associated to the constraints, $u \in \mathbb{R}^{n}$ is the vector of generalized torque inputs, $C(X, \dot{X})$ is the matrix of Coriolis and centripetal forces, $G(X)$ contains conservative forces. $\nabla$ denotes the Euclidean gradient. The impact times will be denoted generically as $t_{k}$ in the following. The admissible domain $\Phi$ is a closed domain in the configuration space where the system can evolve, i.e. $\Phi=\{X \mid F(X) \geq 0\}$. The boundary of $\Phi$ is denoted as $\partial \Phi$. A collision rule is needed to integrate the system in (1) and to render the set $\Phi$ invariant. A collision rule is a relation between the post-impact velocities and the pre-impact velocities. In this work, it is chosen as in [6]:

$$
\begin{gathered}
\dot{X}\left(t_{k}^{+}\right)=-e_{n} \dot{X}\left(t_{k}^{-}\right)+\left(1+e_{n}\right) \arg \min _{z \in T_{\Phi}}\left(X\left(t_{k}\right)\right) \\
\frac{1}{2}\left[z-\dot{X}\left(t_{k}^{-}\right)\right]^{T} M\left(X\left(t_{k}\right)\right)\left[z-\dot{X}\left(t_{k}^{-}\right)\right]
\end{gathered}
$$

where $\dot{X}\left(t_{k}^{+}\right)$is the post impact velocity, $\dot{X}\left(t_{k}^{-}\right)$is the preimpact velocity, $T_{\Phi}(X(t))$ the tangent cone to the set $\Phi$ at $X(t)$ and $e_{n}$ is the restitution coefficient, $e_{n} \in[0,1]$. The restitution mapping in (2) yields a kinetic energy loss at the impact times given by :

$$
\begin{aligned}
T_{L}\left(t_{k}\right) & =-\frac{1}{2} \frac{1-e_{n}}{1+e_{n}}\left[\dot{X}\left(t_{k}^{+}\right)-\dot{X}\left(t_{k}^{-}\right)\right]^{T} M\left(q\left(t_{k}\right)\right) \\
& {\left[\dot{X}\left(t_{k}^{+}\right)-\dot{X}\left(t_{k}^{-}\right)\right] \leq 0 }
\end{aligned}
$$

\subsection{Cyclic task}

In this paper we restrict ourselves to a specific task, or trajectory: a succession of free and constrained phases $\Omega_{k}$. During the transition between a free and a constrained phase, the dynamic system passes into a transition phase $I_{k}$. Transition between constrained and free motion is direct (it suffices that the acceleration $\frac{d^{2}}{d t^{2}}(F(X(t)))$ calculated along solutions of (1) be positive to assure detachment). In the time domain one gets a representation as :

$$
\mathbb{R}^{+}=\Omega_{0} \cup I_{0} \cup \Omega_{1} \cup \Omega_{2} \cup I_{1} \cup \ldots \cup \Omega_{2 k-1} \cup \Omega_{2 k} \cup I_{k} \cup \ldots
$$

where $\Omega_{2 k}$ denotes the time intervals associated to freemotion phases and $\Omega_{2 k+1}$ those for constrained-motion phases. The order of the phases is important but the initial phase may be $\Omega_{0}$ or $I_{0}$ or $\Omega_{1}$.

The items i) and ii) in the introduction imply that the orbit of the desired set $X^{i, n c}(\cdot)$ of the unconstrained system possess the generic form shown in figure 1. It is clear 


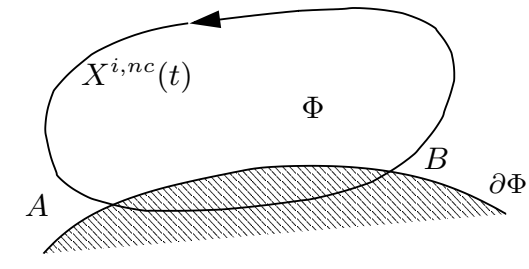

Figure 1: Unconstrained trajectory.

that in particular item ii) implies that $F\left(X^{i, n c}(t)\right)<0$ for some $t\left(\in \Omega_{2 k+1}\right)$, otherwise there would be a zero contact force when the system perfectly tracks the desired motion. Consequently there exists a point $A$ in the configuration space, at which contact is made with $\partial \Phi$. This gives rise to a transition phase. The central issue in the present control problem, is the design of such transition phases. A first idea is to impose a tangential contact, i.e. with $\nabla F\left(X_{d}^{*}\right)^{T} \dot{X}_{d}^{*}=0$, where $X_{d}^{*}(\cdot)$ is a signal entering the control input and playing the role of the desired trajectory during some parts of the motion (the difference between $X_{d}^{*}(\cdot)$, and $X^{i, n c}(\cdot)$ will be made clear below). However

- $\alpha)$ Due to non-zero initial tracking errors $X(0)$ $X_{d}^{*}(0) \neq 0, \dot{X}(0)-\dot{X}_{d}^{*}(0) \neq 0$, impacts may occur.

- $\beta$ ) This is not a robust strategy since a bad estimation of the constraint position, may result to no stabilisation at all on $\partial \Phi$. Consequently it is a much better strategy to impose collisions for stabilisation on $\partial \Phi$.

- $\gamma$ ) In any case, collisions have to be incorporated into the stability analysis.

- $\delta$ ) The best strategy for stabilisation on $\partial \Phi$ is to impose closed-loop dynamics which mimics the bouncing-ball dynamics $\ddot{X}=-g, X \geq 0$ :

$\left.\delta_{1}\right)$ This is very robust with respect to the constraint position uncertainties.

$\delta_{2}$ ) It lends itself very well to Lyapunov stability of some closed-loop Poincaré map.

Secondly, we will see in the next section that the type of stability we desire is based on a single Lyapunov-like function $V(X, \dot{X}, t)$. Then difficulties arise due to the following:

- a) There are non-zero couplings between "tangential" and "normal" coordinates in the inertia matrix $M(X)$ (this will be formulated more rigorously later).

- b) This unique function $V(X, \dot{X}, t)$ has to work for all phases, i.e. for $\Omega_{2 k}$ (ODE), $\Omega_{2 k+1}$ (DAE), and $I_{k}$ (the dynamics may then be seen as a Measure Differential Equation [2]).

- c) If $V \equiv 0$ then any velocity jump $\dot{q}\left(t_{k}^{+}\right) \neq \dot{q}\left(t_{k}^{-}\right)$ implies a positive jump $V\left(t_{k}^{+}\right)-V\left(t_{k}^{-}\right)>0$ in the Lyapunov function. This means that impacts will generally preclude asymptotic stability $\left({ }^{1}\right)$, except in very particular cases of no inertia couplings, in which case things greatly simplify.

- d) The function $V$ has to satisfy $V=0$ when the desired trajectory of the closed-loop system is perfectly tracked, according to the definition of a Lyapunov function. This implies that the desired set of the complete (constrained) system must be used in the definition of $V$.

One therefore realises that the control problem is itself subject to many constraints. The proposed strategy has to cope with these various and sometime antagonist facts (like $\beta$ ) and $\mathbf{c}$ )). Item $\mathbf{c}$ ) hampers the use as time goes to infinity of any controller that would switch at time $t_{s}$ between a free-motion feedback input with $F\left(X_{d}^{*}\left(t_{s}^{-}\right)\right)>$ 0 to a transition phase controller with a "bouncing-ball" dynamics (i.e. such that $\left.F\left(X^{i, n c}\left(t_{s}^{+}\right)\right)<0\right)$. However such a discontinuous input can be used during the transient period. In order to clarify these various notions let us consider a one degree-of-freedom system:

$$
\left\{\begin{array}{l}
\left(\ddot{X}-\ddot{X}_{d}^{*}\right)+\gamma_{2}\left(\dot{X}-\dot{X}_{d}^{*}\right)+\gamma_{1}\left(X-X_{d}^{*}\right)=\lambda \\
0 \leq X \perp \lambda \geq 0 \\
\dot{X}\left(t_{k}^{+}\right)=-e_{n} \dot{X}\left(t_{k}^{-}\right)
\end{array}\right.
$$

where $X_{d}^{*}(\cdot)$ is some twice differentiable function, $\gamma_{2}>0$, $\gamma_{1}>0$. The " $\perp$ " means that $X$ and $\lambda$ are orthogonal, i.e. $X \lambda=0$. It is clear that $X^{i, n c} \equiv X_{d}^{*}$. If $X_{d}^{*}(t)<0$ on some interval of time $I$, then the desired trajectory of the constrained system cannot be $X_{d}^{*}(\cdot)$. Rather, this is going to be simply 0 on $I$. Item $\mathbf{d}$ ) means that the function $V$ used for stability purpose (e.g. a quadratic function of the tracking error) is zero on $\Omega_{2 k+1}$ (constrained-motion phases). Therefore the Lyapunov function will be defined such that on $I_{k}$ and on $\Omega_{2 k+1}$ one has $V(X, \dot{X}, t)=0$. Since this is a tracking control problem and since the desired trajectory is equal to 0 on such phases (even the rebound phase), we conclude that the tracking error $\tilde{X}(\cdot)$ entering $V(\cdot)$ has to satisfy $\tilde{X}(\cdot)=0$, so that $V(\tilde{X}=0, \dot{\tilde{X}}=0)=0$. Thus $\tilde{X}(\cdot)$ cannot be defined from $X_{d}^{*}(\cdot)$ neither from $X^{i, n c}$ but from a third signal which we shall denote as $X_{d}(\cdot)$. Let us again clarify the difference between $X_{d}^{*}(\cdot)$ and $X_{d}(\cdot)$. Let us take a constant $X_{d}^{*}<0$ in (5). Then $X^{i, n c}=X_{d}^{*}$ but since the fixed point of the complementarity system is $(X, \dot{X})=(0,0)$, we must have $V(X=0, \dot{X}=0)=0$ so that the restriction of $V$ to the Poincaré section $\Sigma^{+}=\{X \mid X=0, \dot{X}>0\}$ is a Lyapunov function for the corresponding Poincaré impact mapping $P_{\Sigma}$. Consequently we shall define $X_{d}=0$ during these periods of time. In the following we shall denote $\tilde{X}=X-X_{d}$ and $\bar{X}=X-X_{d}^{*}$. Finally in general $X^{i, n c} \neq X_{d}^{*}$ because $X^{i, n c}$ is the desired set of the closed-loop unconstrained

\footnotetext{
${ }^{1}$ This is mainly due to the fact that the controllers used on phases $\Omega_{k}$ assure asymptotic convergence of the tracking errors towards zero, but do not possess any finite-time convergence properties.
} 
system whereas $X_{d}^{*}$ may be chosen to evolve from one transition phase $I_{k}$ to the next one $I_{k+1}$.

Such conditions appear quite stringent. Actually we are looking for the most direct extension of Lyapunov's second method for complementarity systems as in (1) evolving as in (4). If the task is less complex than (4) and/or the dynamics possess some strong properties (see [2, chapter 8]) then the stability analysis may simplify.

The control strategy which is developed in the sequel, takes all these features into account and especially imposes an desired trajectory $X^{i, n c}$ as depicted in figure 2 . The orbits of the trajectories are depicted. Tangential contact is made at $A^{\prime \prime}$ when force control starts so that $X^{i, n c}$ jumps at $B$. In addition item $\beta$ ) is taken into account by imposing a "bouncing-ball" dynamics only during the transient period, i.e. on $I_{k}$ with $k<+\infty$. In other words the trajectory $X^{i, n c}(t)$ makes a tangential contact with $\partial \Phi$ because if initial data satisfy $X(0)-X_{d}^{*}(0)=0$ and $\dot{X}(0)-\dot{X}_{d}^{*}(0)=0$ on $\Omega_{2 k}$, then $X(t) \equiv X^{i, n c}(t)$ for $t \in \Omega_{2 k}$, but during the transient period the controller assures the existence of collisions on phases $I_{k}$. Therefore between points $A$ and $B^{\prime}$ on figure 2 , one may have $X_{d}^{*}(\cdot)$ which violates the constraint during the transient period, and converges towards a tangential approach trajectory after a finite or infinite number of transition phases (or cycles $\left.\Omega_{2 k} \cup I_{k} \cup \Omega_{2 k+1}\right)$. Between $B$ and $C$ the phase $\Omega_{2 k+1}$ occurs during which objective ii) is fulfilled. The dashed orbit $A A^{\prime} B^{\prime}$ on figure 2 represents $X_{d}^{*}(\cdot)$ during a transition phase with impacts. The system stabilizes on $\partial \Phi$ between $A$ and $B^{\prime}$ when the controller is switched to a force control so that $X^{i, n c}(\cdot)$ and $X_{d}^{*}(\cdot)$ may jump to $B$. In the control scheme described later, the point $B^{\prime}$ will converge (in a finite or infinite number of cycles) towards $A^{\prime \prime}$. We finally define the closed-loop desired trajectory of the complementarity system as $X^{i, c}(\cdot)$. On figure $2, X^{i, c}(\cdot)$ is the curve $\left(C A A^{\prime \prime} C\right)$ and $X^{i, c}(\cdot) \in \partial \Phi$ on $\left(A^{\prime \prime} C\right)$. It is an impactless trajectory. Let us assume that the ultimate goal is to obtain a periodic trajectory to be tracked asymptotically. Then on figure 2 only the orbits of $X^{i, n c}(\cdot)$ (i.e. $A A^{\prime \prime} B C A$ ) and $X^{i, c}(\cdot)$ (i.e. $A A^{\prime \prime} C A$ ) are fixed. The other two orbits may vary from one cycle $\Omega_{2} k \cup I_{k} \cup \Omega_{2 k+1}$ to the next. But on a single phase $I_{k}$ the fixed point of the closed-loop system may indeed be a signal $X_{d} \in \partial \Phi\left(A^{\prime} A^{\prime \prime}\right)$ which differs from $X_{d}^{*} \notin \Phi\left(A^{\prime} B^{\prime}\right)$. The pieces of orbits $\left(A A^{\prime} B^{\prime}\right)$ and the point $A^{\prime}$ generally vary from one cycle $\Omega_{2 k} \cup I_{k} \cup \Omega_{2 k+1}$ to the next cycle $\Omega_{2 k+2} \cup I_{k+1} \cup \Omega_{2 k+3}$.

In summary the control strategy and stability analysis are led with four different trajectories: $X_{d}^{*}(\cdot)$ in the control input, $X_{d}(\cdot)$ in the Lyapunov function, $X^{i, c}(\cdot)$ and $X^{i, n c}(\cdot)$. Still referring to figure 2: when the system is initialised on $X^{i, c}(\cdot)$ between $C$ and $A$ (i.e. on $\Omega_{0}$ ), then $X_{d}(t)=X^{i, c}(t)$ on $\left(A^{\prime \prime} C\right)$ and $X_{d}(t) \in \partial \Phi$ on $\left(A^{\prime \prime} C\right)$. If initially $X(0) \neq X^{i, c}(0)$ and/or $\dot{X}(0) \neq \dot{X}^{i, c}(0)$, then $X_{d}(\cdot)$ differs and is set to zero in the Lyapunov function

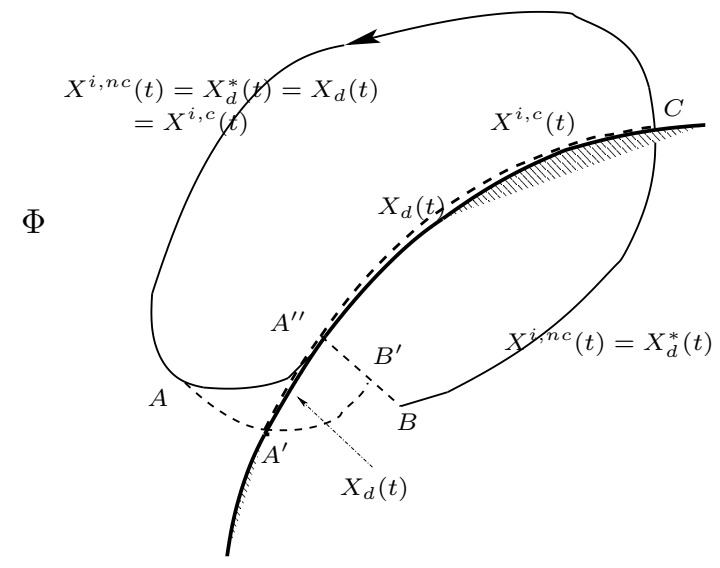

Figure 2: The closed-loop desired trajectories.

at a time corresponding to $A^{\prime}$. This is the major discrepancy compared to unconstrained motion control in which all four trajectories are the same, usually denoted as $X_{d}(\cdot)$.

\section{Stability framework}

The stability criterion used in this paper is an extension of the Lyapunov second method adapted to closed loop mechanical system with unilateral constraints and has been proposed in [3] and [4]. Let $x(\cdot)$ denote the state of the closed-loop system in (1) with some feedback controller $u(X, \dot{X}, t)$.

Definition 1 ( $\Omega$-weakly stable system) The closedloop system is $\Omega$-weakly stable if for each $\epsilon>0$, there exists $\delta(\epsilon)>0$ such that $\|x(0)\| \leq \delta(\epsilon) \Rightarrow\|x(t)\| \leq \epsilon$ for all $t \geq 0, t \in \Omega=\cup_{k \geq 0} \Omega_{k}$. Asymptotic weak stability holds if in addition $x(t) \longrightarrow 0$ as $t \longrightarrow+\infty, t \in \Omega$. Practical weak stability holds if there is a ball centered at $x=0$, with radius $R>0$, and such that $x(t) \in B(0, R)$ for all $t \geq T ; T<+\infty, t \in \Omega, R<+\infty$.

Let us define the closed-loop impact Poincaré map that corresponds to the section $\Sigma_{\mathcal{I}}^{-}=\left\{x \mid F_{i}(X)=\right.$ $\left.0, \dot{X}^{T} \nabla F_{i}(X)<0, i \in \mathcal{I}\right\}$, which is a hypersurface of codimension $\alpha=\operatorname{card}(\mathcal{I})$. The pre-impact velocities are chosen to define $P_{\Sigma_{\mathcal{I}}}$ for a reason given after claim 3. We define:

$$
\begin{aligned}
P_{\Sigma_{\mathcal{I}}}: & \Sigma_{\mathcal{I}}^{-} \rightarrow \Sigma_{\mathcal{I}}^{-} \\
& x_{\Sigma_{\mathcal{I}}}(k) \mapsto x_{\Sigma_{\mathcal{I}}}(k+1) .
\end{aligned}
$$

where $x_{\Sigma_{\mathcal{I}}}$ is the state of $P_{\Sigma_{\mathcal{I}}}$. Let us introduce the positive definite function $V(\cdot)$ that will serve in the subsequent analysis. Let $V_{\Sigma_{\mathcal{I}}}$ denote the restriction of $V$ to $\Sigma_{\mathcal{I}}$.

Definition 2 (Strongly stable system) The system is said strongly stable if: (i) it is $\Omega$-weakly stable, (ii) on phases $I_{k}, P_{\Sigma_{\mathcal{I}}}$ is Lyapunov stable with Lyapunov function $V_{\Sigma_{\mathcal{I}}}$, and (iii) the sequence $\left\{t_{k}\right\}_{k \in \mathbb{N}}$ has a finite accumulation point $t_{\infty}<+\infty$. 
Clearly $P_{\Sigma_{\mathcal{I}}}$ has a fixed point $x_{\Sigma_{\mathcal{I}}}^{*} \in \partial \Phi$. Let us define the jump function $\sigma_{f}(t)=f\left(t^{+}\right)-f\left(t^{-}\right)$and $\lambda[$.] is the Lebesgue measure. Let $V(\cdot)$ satisfy $\beta(\|x\|) \geq V(x, t) \geq$ $\alpha(\|x\|), \alpha(0)=0, \beta(0)=0, \alpha(\cdot)$ and $\beta(\cdot)$ strictly increasing. Let $I_{k}=\left[\tau_{0}^{k}, t_{f}^{k}\right]$.

Claim 1 (Weak Stability [3]) Assume that the task is as in (4), and that

(a) $-\lambda[\Omega]=+\infty$,

(b) - for each $k \in \mathbb{N}, \lambda\left[I_{k}\right]<+\infty$,

(c) $-V\left(x\left(t_{f}^{k}\right), t_{f}^{k}\right) \leq V\left(x\left(\tau_{0}^{k}\right), \tau_{0}^{k}\right)$,

(d) $-V(x(),$.$) uniformly bounded on each I_{k}$.

If on $\Omega, \dot{V}(x(t), t) \leq 0$ and $\sigma_{V}\left(t_{k}\right) \leq 0$ for all $k \geq 0$, then the closed-loop system is $\Omega$-weakly stable. If $\dot{V}(x(t), t) \leq$ $-\gamma(\|x(t)\|), \gamma(0)=0, \gamma(\cdot)$ strictly increasing, then the system is asymptotically $\Omega$-weakly stable.

This accomodates for other types of motions than the one as in (4), see [3]. Let us assume that $t_{\infty}<+\infty$. It is noteworthy that from [1, proposition 4.11] this implies $e_{n}<1$ (because if $e_{n}=1$ impact times satisfy $t_{k+1}-t_{k} \geq$ $\beta_{k}>0$ with $\sum_{k \geq 0} \beta_{k}$ unbounded, so that $\left.t_{\infty}=+\infty\right)$.

Claim 2 (Weak Stability) Let us assume that (a) and (b) in claim (1) and that, in addition

(a) - outside $I_{k}$ one has $\dot{V}(t) \leq-\gamma V(t)$ for some $\gamma>0$,

(b) - inside $I_{k}$ one has $V\left(t_{k+1}^{-}\right)-V\left(t_{k}^{+}\right) \leq 0$, for all $k \geq 0$,

(c) - the system is initialized on $\Omega_{0}$ with $V\left(\tau_{0}^{0}\right) \leq 1$,

(d) $-\sum_{k>0} \sigma_{V}\left(t_{k}\right) \leq K V^{\kappa}\left(\tau_{0}^{k}\right)+\epsilon$ for some $\kappa \geq 0, K \geq 0$ and $\epsilon \geq 0$.

Then there exists a constant $N<+\infty$ such that $\lambda\left[t_{\infty}^{k}, t_{f}^{k}\right]=N$, for all $k \geq 0$ (the cycle index), and such that:

(i) - If $\kappa \geq 1, \epsilon=0$ and $N=\frac{1}{\gamma} \ln \left(\frac{1+K}{\delta}\right)$ for some $0<\delta<1$, then $V\left(\tau_{0}^{k+1}\right) \leq \delta V\left(\tau_{0}^{k}\right)$. The system is asymptotically weakly stable.

(ii) - If $\kappa<1$, then $V\left(\tau_{0}^{k}\right) \leq \delta(\gamma)$, where $\delta(\gamma)$ is a function which can be made arbitrarily small by increasing $\gamma$. The system is practically $\Omega$-weakly stable with $R=$ $\alpha^{-1}(\delta(\gamma))$.

Proof. The proof is omitted for the sake of paper brevity.

Claim 3 (Strong Stability [3]) The system is strongly stable if in addition to the conditions in claim 1 one has:

- $V\left(t_{k+1}^{-}\right) \leq V\left(t_{k}^{-}\right)$

- $V$ is uniformly bounded and time continuous on $I_{k}-$ $\cup_{k}\left\{t_{k}\right\}$.

\section{Tracking controller framework}

\subsection{Controller Structure}

To make the controller design easier the dynamical equations (1) are considered in the generalized coordinates introduced in [5]. After transformation in the new coordinates $q=\left[\begin{array}{c}q_{1} \\ q_{2}\end{array}\right], q_{1}=\left[\begin{array}{c}q_{1}^{1} \\ \vdots \\ q_{1}^{m}\end{array}\right], q=Q(X) \in \mathbb{R}^{n}$, the dynamic system is as follows :

$$
\left\{\begin{array}{l}
M_{11}(q) \ddot{q}_{1}+M_{12}(q) \ddot{q}_{2}+C_{1}(q, \dot{q}) \dot{q}+g_{1}(q)=T_{1}(q) u+\lambda \\
M_{21}(q) \ddot{q}_{1}+M_{22}(q) \ddot{q}_{2}+C_{2}(q, \dot{q}) \dot{q}+g_{2}(q)=T_{2}(q) u \\
q_{1}^{i} \geq 0, \quad q_{1}^{i} \lambda_{i}=0, \quad \lambda_{i} \geq 0,1 \leq i \leq m \\
\text { Collision rule }
\end{array}\right.
$$

where the set of complementarity relations can be written more compactly as $0 \leq \lambda \perp D q \geq 0$ with $D=$ $\left[I_{m}: 0\right] \in \mathbb{R}^{m \times n}, I_{m}$ is the identity matrix. Clearly $M_{21}(q)=M_{12}^{T}(q) \in \mathbb{R}^{(n-m) \times m}, M_{11}(q) \in \mathbb{R}^{m \times m}$, $M_{22}(q) \in \mathbb{R}^{(n-m) \times(n-m)}$. In the new coordinates $q$ one therefore has $\Phi=\{q \mid D q \geq 0\}$. The tangent cone $T_{\Phi}\left(q_{1}=0\right)=\{v \mid D v \geq 0\}$ is the space of admissible velocities on the boundary of $\Phi$.

Let us choose:

$$
V(t, \tilde{q}, \dot{\tilde{q}})=\frac{1}{2} \dot{\tilde{q}}^{T} M(q) \dot{\tilde{q}}+\frac{1}{2} \gamma_{1} \tilde{q}^{T} \tilde{q}
$$

with $\tilde{q}(\cdot)=q(\cdot)-q_{d}(\cdot)$. The control law used in this scheme is based on the controller presented in [7], originally designed for free-motion position and velocity global asymptotic tracking. Let us propose:

$T(q) u=\left\{\begin{array}{l}U_{n c}=M(q) \ddot{q}_{d}^{*}+C(q, \dot{q}) \dot{q}_{d}^{*}+g(q)-\gamma_{1}\left(q-q_{d}^{*}\right) \\ -\gamma_{2}\left(\dot{q}-\dot{q}_{d}^{*}\right) \\ U_{t}=U_{n c} \quad \text { before the first impact } \\ U_{t}=g(q)-\gamma_{1} \bar{q}-\gamma_{2} \dot{q} \text { after the first impact } \\ U_{c}=U_{n c}-P_{d}+K_{f}\left(P_{q}-P_{d}\right)\end{array}\right.$

where $T(q)=\left(T_{1}(q), T_{2}(q)\right)^{T} \in \mathbb{R}^{n \times n}, \gamma_{1}>0, \gamma_{2}>0$, $K_{f}>0, P_{d}$ is the desired force we want for the permanently constraint motion. The signals $q_{d}^{*}, q_{d}$ and $\bar{q}$ will be defined later, as well as the switching conditions between the controllers in (9). The interest for choosing this controller is that the function $V(t, \tilde{q}, \dot{\tilde{q}})$ in $(8)$ is very close to the total energy of the system. Notice that $u$ is independent of the restitution coefficient $e_{n}$. From (9) the third condition in claim 1 can be replaced by $V\left(t_{f}^{k}\right) \leq V\left(t_{0}^{-}\right)$ since $V\left(t_{0}^{-}\right) \leq V\left(\tau_{0}^{k}\right)$.

\subsection{Design of the desired trajectory}

During the transition phase the control signal $q_{d}^{*}(t)$ is defined as follows (see figure 3 for $q_{1 d}^{*}(\cdot)$, where $A, A^{\prime}, B^{\prime}, B$ and $C$ correspond to figure 2 ): 

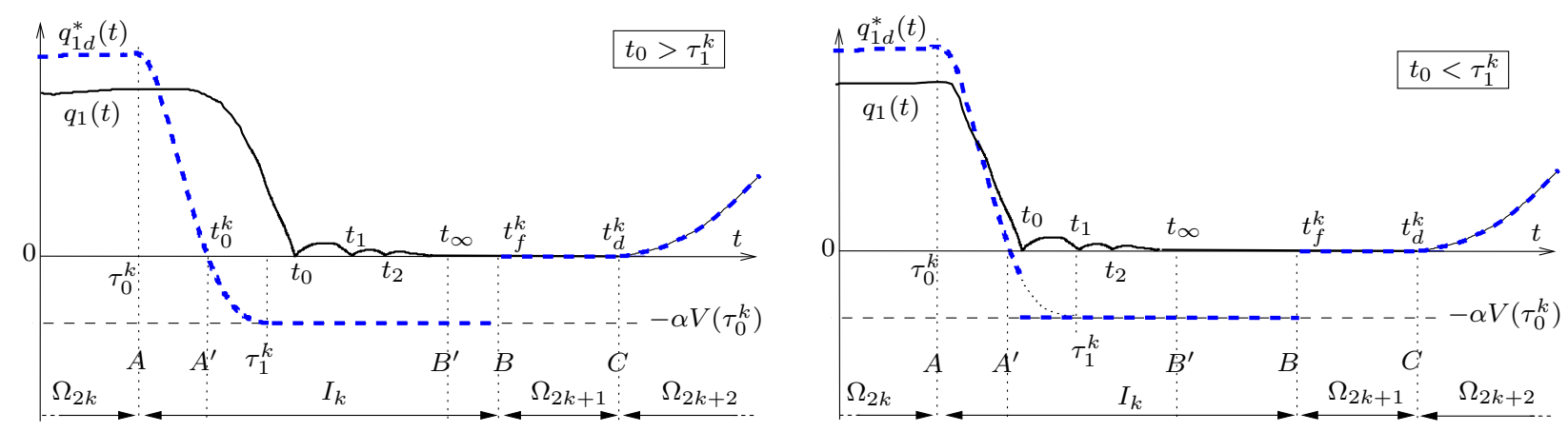

Figure 3: Trajectory $q_{1 d}^{*}(t)$

Let us note that the indices $k$ for the phases $\Omega_{k}$ and $I_{k}$ and for the impact times $t_{k}$, are not related. They are dummy variables. Let us define:

- $\tau_{0}^{k}$ is chosen by the designer as the start of the transition phase,

- $t_{0}^{k}$ is the time corresponding to $q_{1 d}^{*}\left(t_{0}^{k}\right)=0$,

- $t_{0}$ corresponds to the first impact,

- $t_{\infty}$ corresponds to the finite accumulation point of the sequence $\left\{t_{k}\right\}_{k \geq 0}$,

- $t_{f}^{k}$ is the end of the transition phase,

- $\tau_{1}^{k}$ is such that $q_{1 d}^{*}\left(\tau_{1}^{k}\right)=-\alpha V\left(\tau_{0}^{k}\right)$ and $\dot{q}_{1 d}^{*}\left(\tau_{1}^{k}\right)=0$ $\left({ }^{2}\right)$.

- $\Omega_{2 k+1}=\left[t_{f}^{k}, t_{d}^{k}\right]$.

On $\left[\tau_{0}^{k}, t_{0}\right)$, we impose that $q_{d}^{*}(t)$ is twice differentiable, and $q_{1 d}^{*}(t)$ decreases towards $-\alpha V\left(\tau_{0}^{k}\right)$ on $\left[\tau_{0}^{k}, \tau_{1}^{k}\right]$. In order to cope with the coupling between $q_{1}$ and $q_{2}\left(M_{12} \neq\right.$ $0)$, the signal $q_{2 d}^{*}(t) \in C^{2}\left(\mathbb{R}^{+}\right)$is frozen during the transition phase, i.e.:

- $q_{2 d}^{*}(t)=q_{2 d}^{*}, \dot{q}_{2 d}^{*}(t)=0$ on $\left[\tau_{0}^{k}, t_{\infty}\right]$

- $q_{2 d}^{*}(t)$ is defined on $\left[\tau_{0}^{k}, t_{0}^{k}\right]$ such that $\dot{q}_{2 d}^{*}\left(t_{0}^{k}\right)=0$.

On $\left(t_{0}, t_{f}\right]$, we define $q_{d}$ and $q_{d}^{*}$ as follows :

$$
q_{d}=\left(0, q_{2 d}^{*}\right)^{T}, q_{d}^{*}=\left(-\alpha V\left(\tau_{o}^{k}\right), q_{2 d}^{*}\right)^{T}
$$

On $\left[t_{f}^{k}, t_{d}^{k}\right]$ we set $q_{d}=\left[0, q_{2 d}(t)\right]^{T}$. The purpose of $q_{d}^{*}$ is to create a "virtual" potential force which stabilizes the system on $\partial \Phi$ even if the position of the constraint is uncertain. Consequently the fixed point $\left(q_{d}, \dot{q}_{d}\right)$ of the complementarity system is used in the expression of the Lyapunov fonction $\left(\tilde{q}=q-q_{d}\right)$, whereas the unreachable fixed point $q_{d}^{*}$ is used in the control law (in (9) $\bar{q}=q-q_{d}^{*}$ with $q_{d}^{*}$ as in (10)). In $U_{n c}$ in $(9)$ we have $q_{d}^{*}(\cdot)=q_{d}(\cdot)$ since $q_{d}^{*}(t)=q_{d}(t)$ for $t \in \Omega_{2 k} \cup\left[\tau_{0}^{k}, t_{0}\right]$. In summary, after the first impact at $t_{0}, q_{1 d}(\cdot)$ is set to zero while in case $\tau_{1}^{k}>t_{0}$,

\footnotetext{
${ }^{2}$ In [2] [4] it is implicitly assumed in the stability proofs that $\tau_{1}^{k}<t_{0}$, which is a shortcoming that we avoid in this paper.
}

$q_{1 d}^{*}(\cdot)$ is set to $-\alpha V\left(\tau_{0}^{k}\right)$ (in other words $U_{t}$ switches as indicated in (9)). Since $\dot{q}_{1 d}\left(t_{0}^{-}\right) \neq 0$ and $q_{1 d}\left(t_{0}^{-}\right) \neq 0$ in general, the trajectory $q_{1 d}(\cdot)$ behaves like in a sort of plastic collision $\left(e_{n}=0\right)$. With respect to figure 2 , one has $\tau_{0}^{k}$ at $A, t_{\infty}$ at $B^{\prime}, t_{0}$ at $A^{\prime}, t_{d}^{k}$ at $C$, and $B$ at $t_{f}^{k}$ (the term $-P_{d}-K_{f} P_{d}$ defines the signal $X_{d}^{*}(\cdot)$ between $B$ and $C$ on figure 2). If $V\left(\tau_{0}^{k}\right)=0$ then $A^{\prime \prime}$ corresponds to the time $\tau_{1}^{k}$.

The piece of curve $A A^{\prime}$ on figure 2 is normal to $\partial \Phi$ (which in coordinates $q$ is the codimension- $m$ plane $\left.q_{1}=0\right)$. The closed-loop desired trajectory $X^{i, c}(\cdot)$ is defined as $q^{i, c}(t)=$ $q_{d}^{*}(t)$ on $\Omega_{2 k}, q^{i, c}(t)=q_{d}^{*}(t)$ with $\alpha=0$ on $I_{k}$, and $q_{1}^{i, c}(t)=$ 0 on $\Omega_{2 k+1}, q_{2}^{i, c}(t)=q_{2 d}^{*}(t)$ on $\mathbb{R}^{+}$. It is impactless.

\subsection{Closed-loop stability analysis}

The closed-loop state can be chosen as $x=(\tilde{q}, \dot{\tilde{q}})$, according to definition 1 which concerns only phases $\Omega_{k}$.

Definition $3\{C I\}$ is the subspace of initial conditions $x(0)$ which assure $t_{0} \geq \tau_{1}^{k}$ uniformly along a motion as in (4).

Let us assume that $m=1$ now.

Assumption 1 The controller $U_{t}$ in (9) assures that a sequence $\left\{t_{k}\right\}_{k \geq 0}$ of impact times exists, with $\lim _{k \rightarrow+\infty} t_{k}=t_{\infty}<+\infty$.

One difficulty in the stability analysis along a path like in (4), is to assure that initial tracking errors do not increase from one cycle $\Omega_{2 k} \cup I_{k} \cup \Omega_{2 k+1}$ to the next, due to the impacts. One key point in the stability is the value of the first jump of $V($.$) , i.e. \sigma_{V}\left(t_{0}\right)$. One has

$$
\left\{\begin{aligned}
\sigma_{V}\left(t_{k}\right)= & T_{L}\left(t_{k}\right) \leq 0, k \geq 1 \\
\sigma_{V}\left(t_{0}\right)= & T_{L}\left(t_{0}\right)-\frac{1}{2} \gamma_{1} q_{1 d^{2}}\left(t_{0}^{-}\right)-\frac{1}{2} \dot{q}_{d}\left(t_{0}^{-}\right)^{T} M \dot{q}_{d}\left(t_{0}^{-}\right) \\
& +M_{11} \dot{q}_{1}\left(t_{0}^{-}\right) \dot{q}_{1 d}\left(t_{0}^{-}\right)+\dot{q}_{2}\left(t_{0}^{-}\right)^{T} M_{21} \dot{q}_{1 d}\left(t_{0}^{-}\right)
\end{aligned}\right.
$$

It is noteworthy that the equalities in (11) hold independently of the chosen impact rule. The only assumption is that impacts dissipate kinetic energy. The choice for $q_{d}^{*}($.$) and switching strategy, is mainly done to pos-$ sibly obtain $\sigma_{V}\left(t_{k}\right) \leq 0$ for $k \geq 0$. Let us now state the following: 
Claim 4 Let assumption (1) hold. The system defined by (1) in closed-loop with the controller in (9) and $q_{d}(\cdot), q_{d}^{*}(\cdot)$ as defined in section 3.2, is :

(i) - Asymptotically strongly stable if $x(0) \in\{C I\}$.

(ii) - Asymptotically strongly stable if $q_{d}^{*}($.$) is designed such$ that at the first impact time of each phase $I_{k}$ we have $\left[M_{11} \dot{q}_{1}\left(t_{0}^{-}\right)+\dot{q}_{2}\left(t_{0}^{-}\right)^{T} M_{21}\right] \dot{q}_{1 d}^{*}\left(t_{0}^{-}\right) \leq 0$.

(iii) - Asymptotically strongly stable if $M_{12}=0$ and $e_{n}=0$.

(iv) - Asymptotically weakly stable if $M_{12}=0$ and $0 \leq e_{n}<$ 1.

Proof. The proof is omitted for the sake of paper brevity and since it relies on similar arguments as in [4].

\subsection{Perspectives}

The application of claim 2 requires to choose a control law $U_{n c}$ such that item (a) of claim 2 applies. For instance the Slotine and $\mathrm{Li}$ or an input/output feedback linearization can be tried. The crucial step is item (d) of claim 2 .

\section{Simulation example}

This control scheme is tested in simulation on a 2-link planar manipulator for the simplest case of a scalar constraint. The constraint surface corresponds to the ground $(y=0)$. The natural generalized coordinates so that the dynamics fits with (7), with $m=1$, are the work-space coordinates $(x, y)$. We take:

$$
q=\left[\begin{array}{l}
q_{1} \\
q_{2}
\end{array}\right]=\left[\begin{array}{l}
y \\
x
\end{array}\right], y>0
$$

Figure 4 shows the evolution of $q_{1}(t)$ and $q_{2}(t)$ during cyclic tasks as in (4). On the graph of $q_{1}$, the asymptotic convergence of the controller is exhibited as the value of $\alpha V\left(\tau_{0}^{k}\right)$ decreases exponentially. The graph of $q_{2}$ shows the coupling between $q_{1}$ and $q_{2}$. At each impact time a jump in $\dot{q}_{2}$ occurs. The periodic step on $q_{2 d}$ corresponds to the transition phase during which $q_{2 d}$ needs to be frozen.

\section{Conclusion}

This paper deals with the tracking control of fully actuated Lagrangian systems subject to frictionless unilateral constraints. These dynamical systems are named complementarity systems because they involve complementarity conditions. They are nonsmooth because the velocity may possess discontinuities (at impact times), so that the acceleration and the contact force are measures. They may be seen as a complex mixture of ordinary differential equations, differential-algebraic equations, and measure differential equations. The extension of the tracking control of unconstrained (or persistently constrained) Lagrangian systems, towards complementarity Lagrangian systems, is

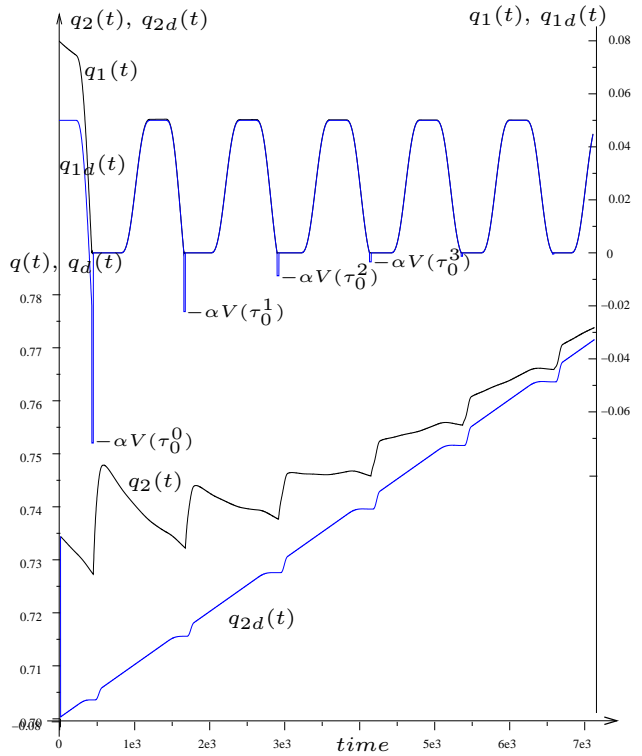

Figure 4: Asymptotic Convergence

not trivial. The aim of this paper is to study the design of a feedback controller for these specific nonsmooth systems, supposed to perform a general cyclic impacting task.

\section{References}

[1] P. Ballard. Formulation and well-posedness of the dynamics of rigid-body systems with perfect unilateral constraints. Phil. Trans. Royal Soc. Lond. A, 359:2327-2346, 2001.

[2] B. Brogliato. Nonsmooth Mechanics. Springer CCES, London, 2nd edition, 1999. Erratum and addenda available at http://www.inrialpes.fr/ bipop/people/brogliato/erratum.ps.

[3] B. Brogliato, S. Niculescu, and P. Orhant. On the control of finite dimensional mechanical systems with unilateral constraints. IEEE Transactions on Automatic Control, 42(2):200-215, February 1997.

[4] B. Brogliato, S.I. Niculescu, and M.D.P MonteiroMarques. On tracking control of a class of complementary-slackness mechanical systems. Systems and Control Letters, 39(4):255-266, March 2000.

[5] N.H. McClamroch and D. Wang. Feedback stabilization and tracking of constrained robots. IEEE Transactions on Automatic Control, 33(5):419-426, May 1988.

[6] J.J. Moreau. Unilateral contact and dry friction in finite freedom dynamics. In Nonsmooth Mechanics and Applications, CISM Courses and Lectures no 302. Springer-Verlag, 1988.

[7] B. Paden and R. Panja. Globally asymptotically stable $\mathrm{pd}+$ controller for robot manipulators. Int. J. Control, 47:1697-1712, 1988. 